\title{
Evidence synthesis based on non-randomised studies-a critical review of studies leading to conclusions on fall risk properties of loop diuretics/beta-blockers
}

\author{
Susanna M. Wallerstedt ${ }^{1,2}$ (D) Mikael Hoffmann $^{3}$ (D) \\ Received: 4 July 2019 / Accepted: 1 September 2019 / Published online: 10 October 2019 \\ (C) The Author(s) 2019
}

\begin{abstract}
Purpose To describe methodological and reporting issues in non-randomised comparative drug safety studies pooled in metaanalyses, with focus on confounding by indication.

Methods All studies included in statistically significant meta-analyses in a recent publication investigating fall risk properties of cardiovascular drugs were reviewed. Study characteristics were extracted and assessed.

Results Nine studies, including between 498 and 321,995 individuals, contributed data to the significant meta-analyses in which loop diuretics and beta-blockers were associated with falls, five published in 2015. Five individual studies reported a statistically significant association. In the five cohort studies, characteristics of exposed vs unexposed individuals were either not reported $(n=3)$ or differed substantially regarding morbidity $(n=2)$. Drug treatment was determined at baseline, and data on falls were collected for up to 2 years thereafter. Out of the four case-control studies, the cases and controls in only one study were matched for morbidity. Morbidity characteristics of fallers compared with non-fallers were either not reported $(n=2)$ or they differed $(n=$ 1 ) or were reported according to the matched-for diseases $(n=1)$. Confounding by indication was explicitly discussed in two studies. None of the abstract conclusions considered causality issues or the possibility of confounding by indication.

Conclusions Confounding by indication is a major issue in non-randomised comparative drug safety studies, a problem which may be concealed in meta-analyses. To enhance such research, compared groups need to be balanced regarding relevant factors including morbidities and characteristics adequately reported. Confounding by indication needs to be explicitly discussed and highlighted in the abstract conclusion.
\end{abstract}

Keywords Drug safety - Cardiovascular drugs - Confounding by indication - Evidence-based medicine $\cdot$ Falls · Pharmacoepidemiology

\section{Introduction}

The basis for prescribing decisions is a medical assessment that the expected benefits of a drug exceed the risks for the specific patient, given their health condition. Therefore, the underlying evidence regarding benefits and harms, often summarised in systematic reviews and meta-analyses, is

Susanna M. Wallerstedt

susanna.wallerstedt@pharm.gu.se

1 Department of Pharmacology, Sahlgrenska Academy, University of Gothenburg, Box 431, SE-405 30 Gothenburg, Sweden

2 HTA Centre, Sahlgrenska University Hospital, Gothenburg, Sweden

3 The NEPI Foundation - Swedish Network for

Pharmacoepidemiology, Linköping University, Linköping, Sweden crucial in the process of prescribing. If the benefits are overestimated, patients will unjustifiably be put at risk. If, on the other hand, the risks are overstated, physicians may be guided to refrain from prescribing and the patients may not receive the benefits of treatment. Beneficial effects are generally well established through randomised controlled trials (RCTs), and meta-analyses of such studies are a standard procedure for evidence synthesis. Regarding safety aspects of drugs, not detected in RCTs, evidence often has to be based on non-RCTs using epidemiological methods, i.e. pharmacoepidemiological studies. When such studies are also pooled in meta-analyses, this may imply a considerably greater risk of bias, in particular because of selection bias and confounding by indication. Indeed, drug treatment is not random as physicians and, to a certain degree, patients make active and informed decisions. 
As clinical pharmacologists educating health care personnel and contributing as experts in pharmacotherapeutic contexts [1-3], we occasionally encounter the belief that fall risk properties of specific cardiovascular drugs are a major concern. Two previous systematic reviews published in 1999 [4] and 2009 [5] may have contributed to this belief, cited in 481 and 638 publications, respectively (Scopus, August 2019). Indeed, there are general recommendations against prescribing cardiovascular drugs because of fall risk properties, for example in sets of indicators of prescribing quality for older patients $[6,7]$. A third, and the most recent, systematic review with meta-analyses evaluating cardiovascular drugs as risk factors for falls was published in 2018 [8]. It concluded that loop diuretics are associated with a $36 \%$ increased risk of falls and beta-blockers with a $12 \%$ decreased risk.

Confounding by indication has recently been reported to be the most prevalent methodological problem in pharmacoepidemiological research [9]. Meta-analyses that led to conclusions regarding associations between cardiovascular drugs and falls provide an opportunity to systematically take a deeper look at methodological and reporting issues, including confounding by indication, in current comparative drug safety research that contributes to prescribing recommendations. We performed the present study with the aim to elucidate these issues and to provide informed suggestions to enhance future research within the field.

\section{Methods}

In this study, we reviewed all publications contributing data to statistically significant meta-analyses in the most recent of the systematic reviews investigating cardiovascular drugs as a risk factor for falls, that is, those concerning loop diuretics and beta-blockers [8]. We extracted study characteristics and assessed methodological as well as reporting issues with a focus on aspects related to confounding by indication. We also recorded the impact factor of the scientific journals during the year in question, retrieved from the Journal Citation Index.

One author (S.M.W.) extracted data from the studies, and the other author (M.H.) independently checked these. Data extraction included the study design (retrieved from the meta-analysis [8]), the data sources used, characteristics of the individuals analysed, recording of drug exposure and collection of outcome data as well as information on the methodology used regarding matching and adjustments. Further, the results for loop diuretics and beta-blockers were extracted.

To shed light on issues related to confounding by indication, we recorded if morbidities of compared groups were presented according to the design. If presented, we assessed whether the compared groups differed regarding morbidity. We also determined whether confounding by indication was highlighted in the abstract and in the discussion section and whether causality issues were considered. Finally, we recorded whether clinical advice was provided based on the results and whether there was a discussion on the benefit-risk balance of drug treatment.

The assessments were discussed by the authors in an iterative process where one author provided assessments and suggestions for classifications, and the other checked these and contributed with further information and suggested changes to the classifications. Disparities were resolved by discussion and consensus was reached.

A descriptive analysis of the data was performed. Based on the findings, we summarised suggestions to guide future research within the field.

\section{Results}

A total of nine studies were included in the review. In all, 60 individuals authored the publications which were each written by two to 17 authors. The impact factor of the journals in the year in which the studies were published ranged from 2.23 [10] to 6.35 [11].

In Table 1, characteristics of the studies are presented. The studies included 498 to 321,995 individuals, whose data were extracted either from registers $(n=3)$, from another study $(n=$ 4 ) or were collected for the purpose of evaluating fall risk properties of drugs $(n=2)$. The patients came either from a general population sample $(n=2)$ or were nursing home residents $(n=2)$, inpatients $(n=1)$, older people with ability to walk $(n=3)$ or individuals who had experienced an ischaemic cerebrovascular event $(n=1)$. The mean age of the patients in the studies ranged between 70 and 84 years.

Five studies had a cohort design while the remaining four were case-control studies (Table 2). In the cohort studies, characteristics of exposed vs unexposed individuals were either not reported $(n=3)$ or differed substantially regarding morbidity $(n=2)$. In one of the latter studies, age also differed between the comparison groups [15]. In all case-control studies, the controls were matched for age and gender. Cases and controls were matched for morbidity in only one of the casecontrol studies. Morbidity characteristics between fallers and non-fallers were either not reported $(n=2)$ or differed $(n=1)$, or were reported according to the matched-for diseases $(n=1)$.

In the cohort studies, drug exposure was determined only at baseline, and falls were recorded during a follow-up period of 6 months to 2 years. All studies then applied a cross-sectional analysis. In three of the case-control studies, drug use was estimated from prescribed/dispensed drugs 30-60 days before the index date; in the fourth, the prescribed drugs in the medical records on the day of falling were used for the estimation.

Five studies reported a statistically significant association between loop diuretics and/or beta-blockers and falls (Table 1). In two of these, morbidities in the compared groups were 


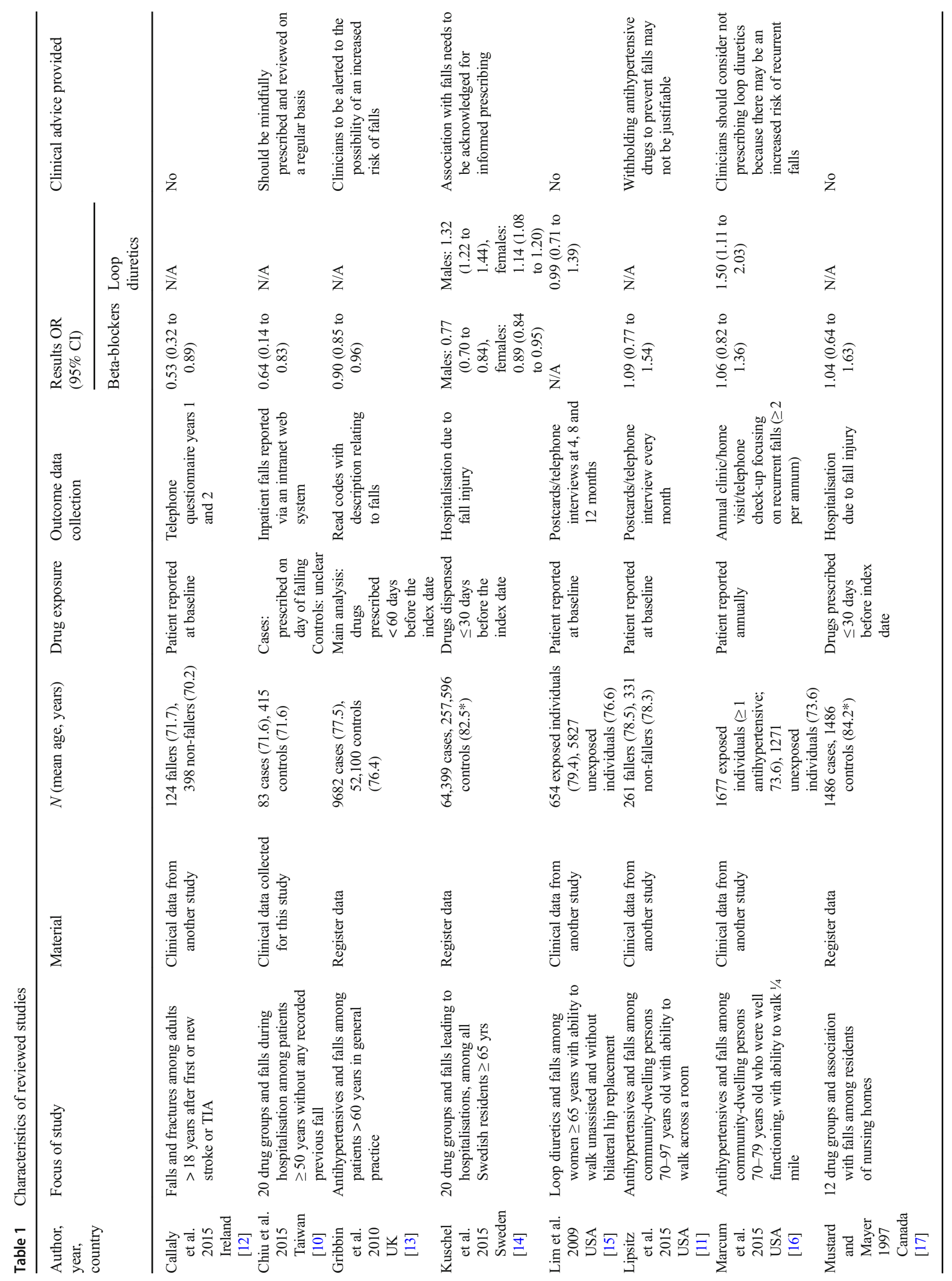




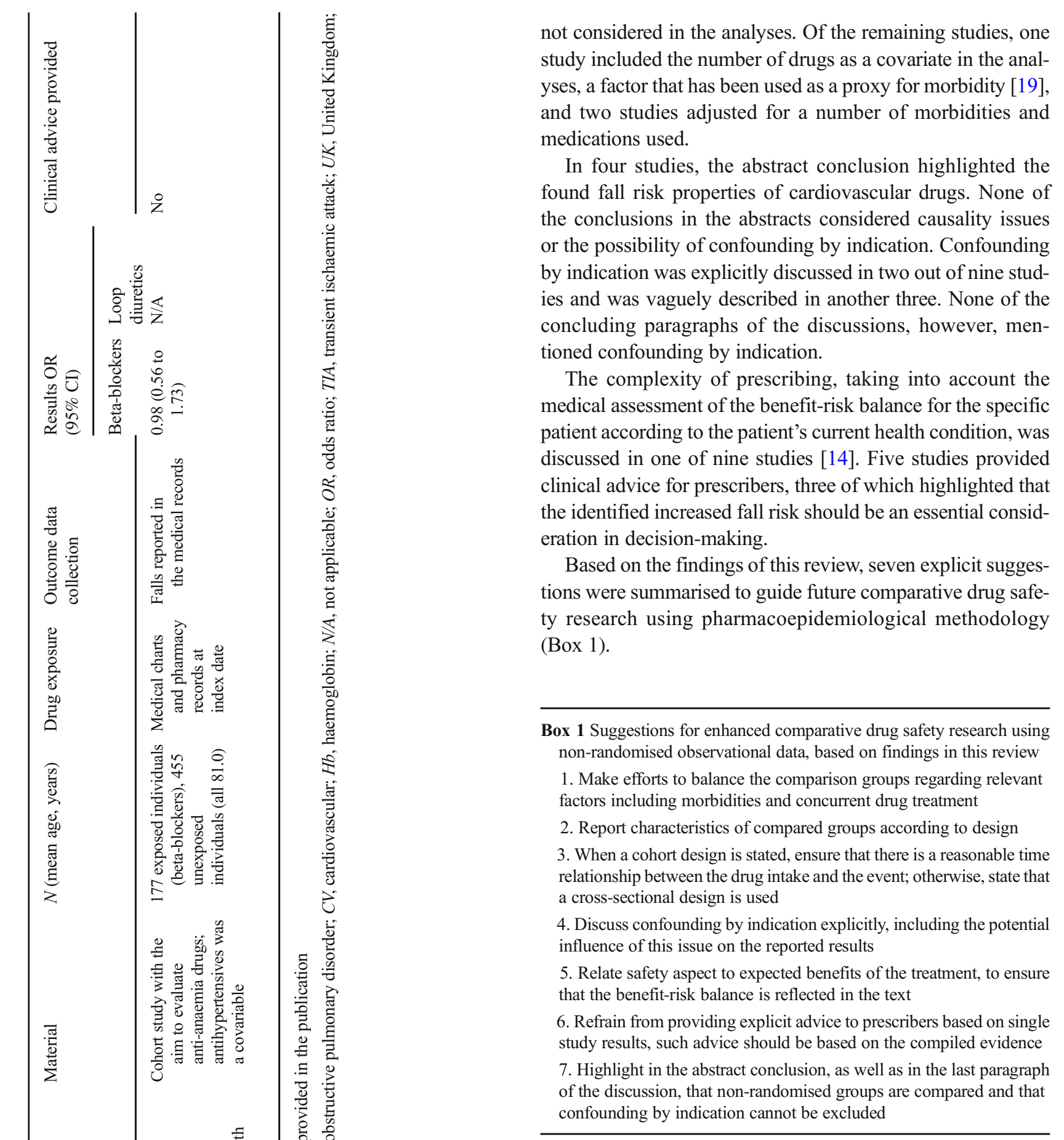

\section{Discussion}

In this review, we found that studies leading to the conclusion in a current meta-analysis of non-RCTs, that loop diuretics increase and beta-blockers decrease the risk of falls, had major methodological problems. For example, morbidity was not considered at all in two studies and only marginally in the rest. Further, characteristics of the compared groups were often inadequately described, severely limiting the potential for 
interpretation of the findings. In addition, drug exposure was often not adequately handled; the possibility of confounding by indication was discussed in only a few studies; and causality considerations were not mentioned in any abstract.

The implications of our findings are serious as metaanalyses have an important role in evidence-based decisionmaking. Indeed, the term meta-analysis is connected to a high level of evidence. However, this is true only if based on RCTs without serious biases. Meta-analyses on non-RCTs, on the other hand, may be controversial. If aggravating issues in non-RCTs are not visible in the pooled results, there may be room for misinterpretations and hasty conclusions. In fact, the study that most influenced the results in the significant metaanalyses of loop diuretics and beta-blockers, because of its large study size, did not consider morbidity in the analyses other than to give, in numerical categories, the number of unique pharmacological subgroups (Anatomic Therapeutic Chemical classification (ATC), level 3 [20]), dispensed over a 120-day period from pharmacies [14]. One may speculate, based on medical common sense, that the results of the metaanalyses could be explained by prescribing practices, with betablockers being prescribed to those in better health and loop diuretics to those in worse. We have not reviewed the studies contributing to previous meta-analyses on associations between cardiovascular drugs and falls $[4,5]$, but they were all based on observational data with risks of confounding by indication.

An important aspect when investigating the effects of drugs is that there needs to be a reasonable time relationship between drug intake and reaction. Although studies with a cohort design are acknowledged to allow conclusions on causality, the potential to learn about fall risk properties of drugs may diminish when, as in this review, exposure is estimated at baseline and information on falls is collected for up to 2 years after baseline. Under these circumstances, it may be more informative to state that a cross-sectional design has been applied, thereby minimising the risk of misinterpreting causality.

Interestingly, three studies advised physicians to consider the fall risk increasing properties of drugs before prescribing. Given that all drugs have benefits and harms and that our review suggests that fall risk may be overstated because of methodological problems, it ought to be emphasised that one-sided attention to harms could contribute to withholding valuable treatment from patients. Still, all but one publication lacked a discussion on the benefit-risk balance of drug treatment. Although safety issues may require a less solid base of evidence, according to the ethical principle not to harm others and also the better safe than sorry principle, providing prescribing advice based on biased evidence implies risks. If a patient on antihypertensive drugs is orthostatic or their blood pressure is too low, it would probably not be controversial to suggest that these drugs should be withdrawn or the dose should be adjusted because the benefit-risk balance would not be expected to be positive for that specific patient. However, when a drug group is said to cause falls without putting this within the context of clinical practice or science, readers may be confused and make hasty conclusions. Indeed, from a medical perspective, it is surprising that none of the studies provided information on blood pressure in the comparison groups.

In a time-pressured medical practice, reading of scientific articles may be limited to reading the abstract conclusion and the summarising paragraph at the end of the discussion. Therefore, our finding that these sections of the studies did not include important aspects for interpretation is somewhat disappointing. Although the limitations may be adequately discussed in some cases, the average reader may miss them. Similarly, readers of systematic reviews may miss cautions raised in the discussion if these are not also highlighted in the abstract conclusion. Indeed, the authors of the meta-analysis appraised in this review acknowledged in the discussion, but not in the conclusion of the abstract, that residual confounding by indication could not be ruled out as this was only partly addressed in the included studies [8]. To further facilitate interpretation, one may also consider requiring information on the type of studies pooled in the title, labelling the study as a nonRCT meta-analysis as opposed to an RCT meta-analysis.

To enhance the standards of comparative drug safety research, we provide suggestions regarding methodological and reporting issues (Box 1). Propensity score matching represents one technique to reduce the risk of confounding. Although one study used propensity scores as a covariate in a sensitivity analysis [15], none of the reviewed studies used this matching technique. According to findings of drug effectiveness studies [21], propensity score matching seems to have undergone limited divergence within the research on drug safety. Further, sensitivity analyses may be a valuable tool in pharmacoepidemiological research. One of the reviewed studies performed a sensitivity analysis in which antidepressants were used as a positive control [13]. As far as we are aware, however, the studies contributing to meta-analyses suggesting fall risk properties of antidepressants have not been reviewed. Therefore, it cannot be excluded that biases may have contributed to the significant findings on that drug group as well. Indeed, the association between antidepressants and fractures, a severe result of a fall, has recently been questioned [22].

\section{Strengths and limitations}

The most important strength of this review is that it highlights pertinent methodological and reporting problems within comparative drug safety research based on non-randomised observational data, providing illustrative examples that may contribute to misleading results in meta-analyses [23, 24]. In addition, explicit suggestions for enhanced performance of such research are provided. Another strength of the present study is that it starts off from a recent review of fall risk properties of cardiovascular drugs, thereby reflecting current practice. Furthermore, the assessments were performed by two clinical 


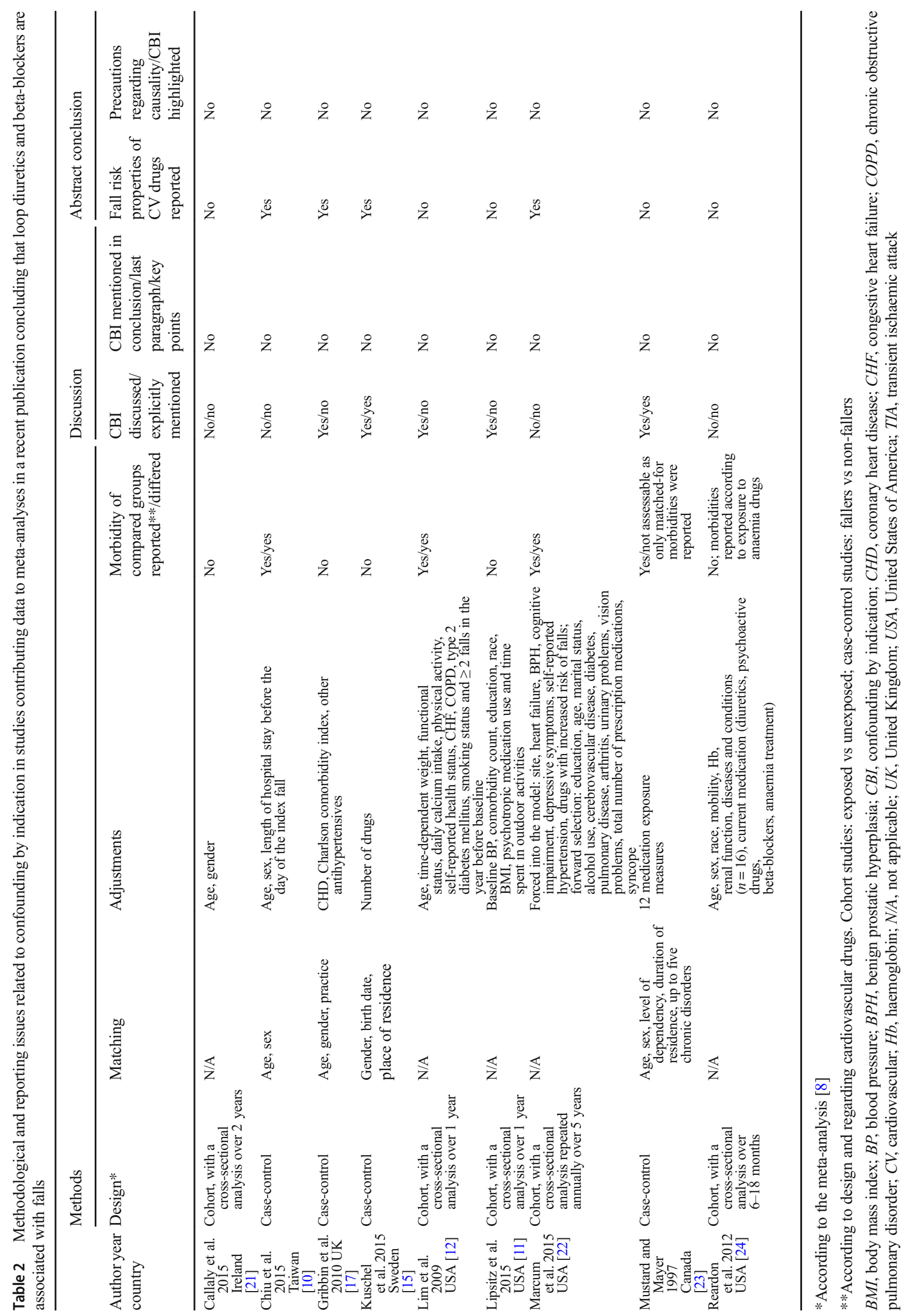


pharmacologists knowledgeable in pharmacoepidemiology and with experience in evidence-based decision-making, both at the patient and at the societal level.

In the review, we chose to focus on cardiovascular drugs with a statistically significant association with falls, according to the meta-analysis. This may be regarded as a limitation affecting the generalisability of the results. Indeed, it cannot be excluded that the reviewed studies represent the better research in the field as they were all published in established journals with acceptable impact factors. Further, they were assessed to be of high or intermediate quality in the original systematic review [8]. Nevertheless, this approach was sufficient to highlight common pitfalls in comparative drug safety research.

\section{Conclusion}

This review illustrates that methodological and reporting issues are prevalent in comparative drug safety studies using nonrandomised observational data, especially regarding confounding by indication. Although seemingly clear and informative, metaanalyses of non-RCTs may bear a risk to conceal such shortcomings. Increased awareness of these issues among researchers is crucial to enhance research within the field and caution recommended when results are pooled in meta-analyses. Authors of pharmacoepidemiological studies are encouraged to explicitly discuss and highlight in the abstract methodological limitations such as confounding by indication when non-randomised data are used to investigate outcomes of drug treatment.

Acknowledgements Open access funding provided by University of Gothenburg.

Author's contributions S.M.W. conceived the study. S.M.W. and M.H. designed the study and performed the assessments. S.M.W. performed the analyses and drafted the manuscript. M.H. revised the manuscript for intellectual content.

Funding information The study was funded by the Swedish Research Council, the Swedish state under the agreement between the Swedish government and the county councils (the ALF-agreement) and the NEPI foundation - Swedish Network for Pharmacoepidemiology. The funding sources did not influence the design, methods, data collection and analysis, preparation of the paper or decision to submit the paper for publication.

Data Availability All data analysed during the current study are provided in the article.

\section{Compliance with ethical standards}

Ethical approval No ethics approval was required as no sensitive data were handled.

Competing interests The authors declare that they have no competing interests.
Open Access This article is distributed under the terms of the Creative Commons Attribution 4.0 International License (http:// creativecommons.org/licenses/by/4.0/), which permits unrestricted use, distribution, and reproduction in any medium, provided you give appropriate credit to the original author(s) and the source, provide a link to the Creative Commons license, and indicate if changes were made.

\section{References}

1. Wallerstedt SM, Rosenborg S (2013) Characteristics and apprehensions of clinical pharmacologists in Swedish healthcare-a questionnaire study. Eur J Clin Pharmacol 69(Suppl 1):95-99. https://doi. org/10.1007/s00228-013-1485-9

2. Birkett D, Brösen K, Cascorbi I, Gustafsson LL, Maxwell S, Rago L, Rawlins M, Reidenberg M, Sjöqvist F, Smith T, Thuerman P, Walubo A, Orme M, Sjöqvist F (2010) Clinical pharmacology in research, teaching and health care: considerations by IUPHAR, the International Union of Basic and Clinical Pharmacology. Basic Clin Pharmacol Toxicol 107(1):531-559. https://doi.org/10.1111/j. 1742-7843.2010.00602.x

3. Aronson JK (2012) What do clinical pharmacologists do? A questionnaire survey of senior UK clinical pharmacologists. Br J Clin Pharmacol 73(2):161-169. https://doi.org/10.1111/j.1365-2125. 2011.04079.x

4. Leipzig RM, Cumming RG, Tinetti ME (1999) Drugs and falls in older people: a systematic review and meta-analysis: II. Cardiac and analgesic drugs. J Am Geriatr Soc 47(1):40-50

5. Woolcott JC, Richardson KJ, Wiens MO, Patel B, Marin J, Khan KM, Marra CA (2009) Meta-analysis of the impact of 9 medication classes on falls in elderly persons. Arch Intern Med 169(21):19521960. https://doi.org/10.1001/archinternmed.2009.357

6. National Board of Health and Welfare (2017) Indicators for appropriate pharmacotherapy in older people [Indikatorer för god läkemedelsterapi hos äldre]. 2017-26-7

7. O'Mahony D, O'Sullivan D, Byrne S, O'Connor MN, Ryan C, Gallagher P (2015) STOPP/START criteria for potentially inappropriate prescribing in older people: version 2. Age Ageing 44(2): 213-218. https://doi.org/10.1093/ageing/afu145

8. de Vries M, Seppala LJ, Daams JG, van de Glind EMM, Masud T, van der Velde N (2018) Fall-risk-increasing drugs: a systematic review and meta-analysis: I. Cardiovascular drugs. J Am Med Dir Assoc 19(4):371.e371-371.e379. https://doi.org/10.1016/j.jamda. 2017.12.013

9. Prada-Ramallal G, Takkouche B, Figueiras A (2019) Bias in pharmacoepidemiologic studies using secondary health care databases: a scoping review. BMC Med Res Methodol 19(1):53. https:// doi.org/10.1186/s12874-019-0695-y

10. Chiu MH, Lee HD, Hwang HF, Wang SC, Lin MR (2015) Medication use and fall-risk assessment for falls in an acute care hospital. Geriatr Gerontol Int 15(7):856-863. https://doi.org/10. 1111/ggi.12359

11. Lipsitz LA, Habtemariam D, Gagnon M, Iloputaife I, Sorond F, Tchalla AE, Dantoine TF, Travison TG (2015) Reexamining the effect of antihypertensive medications on falls in old age. Hypertension 66(1):183-189. https://doi.org/10.1161/ hypertensionaha.115.05513

12. Callaly EL, Ni Chroinin D, Hannon N, Sheehan O, Marnane M, Merwick A, Kelly LA, Horgan G, Williams E, Harris D, Williams D, Moore A, Dolan E, Murphy S, Kelly PJ, Duggan J, Kyne L (2015) Falls and fractures 2 years after acute stroke: the North Dublin population stroke study. Age Ageing 44(5):882-886. https://doi.org/10.1093/ageing/afv093

13. Gribbin J, Hubbard R, Gladman JR, Smith C, Lewis S (2010) Risk of falls associated with antihypertensive medication: population- 
based case-control study. Age Ageing 39(5):592-597. https://doi. org/10.1093/ageing/afq092

14. Kuschel BM, Laflamme L, Moller J (2015) The risk of fall injury in relation to commonly prescribed medications among older peoplea Swedish case-control study. Eur J Pub Health 25(3):527-532. https://doi.org/10.1093/eurpub/cku120

15. Lim LS, Fink HA, Blackwell T, Taylor BC, Ensrud KE (2009) Loop diuretic use and rates of hip bone loss and risk of falls and fractures in older women. J Am Geriatr Soc 57(5):855-862. https:// doi.org/10.1111/j.1532-5415.2009.02195.x

16. Marcum ZA, Perera S, Newman AB, Thorpe JM, Switzer GE, Gray SL, Simonsick EM, Shorr RI, Bauer DC, Castle NG, Studenski SA, Hanlon JT (2015) Antihypertensive use and recurrent falls in community-dwelling older adults: findings from the health $\mathrm{ABC}$ study. J Gerontol A Biol Sci Med Sci 70(12):1562-1568. https:// doi.org/10.1093/gerona/glv095

17. Mustard CA, Mayer T (1997) Case-control study of exposure to medication and the risk of injurious falls requiring hospitalization among nursing home residents. Am J Epidemiol 145(8):738-745

18. Reardon G, Pandya N, Bailey RA (2012) Falls in nursing home residents receiving pharmacotherapy for anemia. Clin Interv Aging 7:397-407. https://doi.org/10.2147/cia.s34789

19. Brilleman SL, Salisbury C (2013) Comparing measures of multimorbidity to predict outcomes in primary care: a cross sectional study. Fam Pract 30(2):172-178. https://doi.org/10. 1093/fampra/cms060

20. WHO Collaborating Centre for (2018) Drug statistics methodology guidelines for ATC classification and DDD assignment 2019, Oslo, Norway

21. Wallerstedt SM, Hoffmann M (2017) Evaluating beneficial drug effects in a non-interventional setting: a review of effectiveness studies based on Swedish prescribed drug register data. Br J Clin Pharmacol 83(6):1309-1318. https://doi.org/ 10.1111/bcp.13206

22. Brännstrom J, Lövheim H, Gustafson Y, Nordstrom P (2019) Association between antidepressant drug use and hip fracture in older people before and after treatment initiation. JAMA Psychiatry 76(2):172-179. https://doi.org/10.1001/jamapsychiatry. 2018.3679

23. Gurevitch J, Koricheva J, Nakagawa S, Stewart G (2018) Metaanalysis and the science of research synthesis. Nature 555(7695): 175-182. https://doi.org/10.1038/nature25753

24. Ioannidis JP (2016) The mass production of redundant, misleading, and conflicted systematic reviews and meta-analyses. Milbank Q 94(3):485-514. https://doi.org/10.1111/1468-0009.12210

Publisher's note Springer Nature remains neutral with regard to jurisdictional claims in published maps and institutional affiliations. 